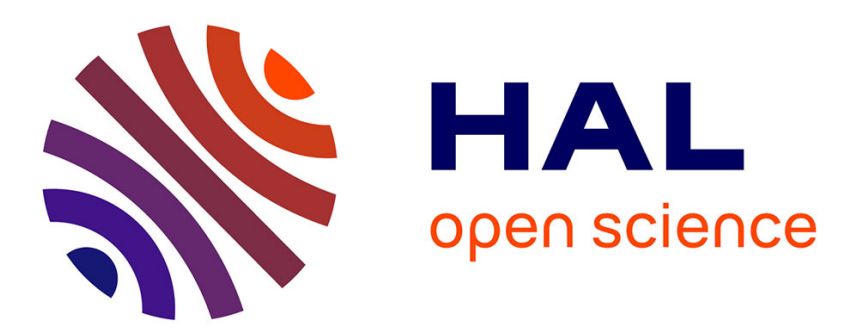

\title{
Adaptive Trajectory Following for a Fixed-Wing UAV in Presence of Crosswind
}

Alexandru Cornel Brezoescu, Tadeo Espinoza, Pedro Castillo, Rogelio Lozano

\section{To cite this version:}

Alexandru Cornel Brezoescu, Tadeo Espinoza, Pedro Castillo, Rogelio Lozano. Adaptive Trajectory Following for a Fixed-Wing UAV in Presence of Crosswind. Journal of Intelligent and Robotic Systems, 2013, 69 (Issue 1-4), pp.257-271. 10.1007/s10846-012-9756-8 . hal-00776093

\section{HAL Id: hal-00776093 https://hal.science/hal-00776093}

Submitted on 15 Jan 2013

HAL is a multi-disciplinary open access archive for the deposit and dissemination of scientific research documents, whether they are published or not. The documents may come from teaching and research institutions in France or abroad, or from public or private research centers.
L'archive ouverte pluridisciplinaire HAL, est destinée au dépôt et à la diffusion de documents scientifiques de niveau recherche, publiés ou non, émanant des établissements d'enseignement et de recherche français ou étrangers, des laboratoires publics ou privés. 


\title{
Adaptive trajectory following for a fixed-wing UAV in presence of crosswind
}

\author{
A. Brezoescu - T. Espinoza - P. Castillo • \\ R. Lozano
}

Received: date / Accepted: date

\begin{abstract}
An adaptive backstepping approach to obtain directional control of a fixed-wing UAV in presence of unknown crosswind is developed in this paper. The dynamics of the cross track error with respect to a desired trajectory is derived from the lateral airplane equations of motion. Adaptation laws are proposed to estimate the parameters of the unknown disturbances and are employed in closedloop system. The stability analysis is proved using Lyapunov theory. In addition, several simulations taking into account unknown wind gusts are performed to analyze the behavior and the robustness of the control scheme. A test platform has been developed in order to validate the proposed control law.
\end{abstract}

Keywords Adaptive control · Fixed-wing UAV · Lyapunov stability

\section{Introduction}

Unmanned Aerial Vehicles (UAVs) represent an area of great interest in the automatic control community. The absence of the pilot renders them best suited to solve dangerous situations. However, it requires significant attention in the flight control design since the vehicle may experience large parameter variations and external disturbances. The largest use of the UAVs is within military applications but they are also used in a growing number of civil applications such as firefighting, digital mapping or monitoring. To increase the usefulness of UAVs, the capability of the autonomous controller to track a reference path is essential. Moreover, the robustness with respect to environmental disturbances must be considered. For example, small UAVs are significantly sensitive to wind since its magnitude may be comparable to the UAVs speed.

A. Brezoescu, P. Castillo and R. Lozano

Université de Technologie de Compiègne, Heudiasyc Laboratory, UMR 7253, France

E-mail: (cbrezoes; castillo; rlozano)@hds.utc.fr

T. Espinoza

División de Estudios de Posgrado e Investigación, ITL, Torreón, Coahuila, México

E-mail: tadeo1519@hotmail.com 
A wide range of trajectory tracking controllers for autonomous vehicles could be found in literature. In [1], the authors addressed the problem of trajectory tracking as a gain scheduling control problem. The proposed methodology was illustrated for an autonomous underwater vehicle that was scheduled on yaw rate and path angle. A nonlinear design was obtained from the interpolation of six linear controllers computed for different values of the gain scheduling variables. The problem of external disturbances was not addressed in this paper.

In gain scheduling theory the system dynamics are considered slowly varying [2][3] which reduces the flight capabilities of an airplane. Trajectory linearization control (TLC) was used in [4] to avoid the use of gain scheduling and to enable operation across the full flight-envelope for a $6 \mathrm{DoF}$ fixed-wing aircraft model. The controller design combined dynamic inversion of the nonlinear equations of motion, to generate nominal force and torque commands, with a linear time varying tracking error regulator to account for model uncertainty. Simulations results were presented for a climbing, bank-to-turn maneuver.

A method based on the vector field approach was proposed in [5] for the case where the time dimension of the reference trajectory is removed. Path following was achieved for straight-lines and circular arcs and orbits in the presence of constant wind disturbances. The algorithm was validated through simulations and real flight tests of a fixed-wing miniature air vehicle.

When accurate knowledge of the vehicle dynamics is not available, adaptive control design can be employed in order to estimate the uncertain parameters. Many of the results in adaptive control are derived from Lyapunov stability theory [6]-[9]. Several flight control algorithms which combines adaptation with other control tools, such as backstepping, neural networks or sliding mode control, can be found in the literature. For instance in [10], flight control laws for two different control objectives were designed employing backstepping technique: maneuvering purpose and automatic control for the flight path angle. Also, two schemes based on adaptive backstepping and nonlinear observer design were proposed for estimating model errors. The proposed controllers were evaluated through simulations.

Likewise, a Lyapunov-based adaptive backstepping approach with online estimation of the uncertain aerodynamic forces and moments was used in [11] to design a flight-path controller for a nonlinear high-fidelity F-16 model. It was shown that trajectory control can still be accomplished with these uncertainties while good tracking performance is maintained. On the other hand, in [12] the authors introduced the design of an adaptive backstepping controller for longitudinal flight-path control when the aerodynamic coefficients are not known exactly. The system followed references in velocity and flight path angle and showed good performance in simulations.

Even if there are many adaptive approaches to flight control design, only few have been developed to realize airplane directional control in presence of unknown wind gusts. The goal of this work is to stabilize an airplane under crosswind and to realize the convergence to zero of the cross track error with respect to a desired trajectory. Moreover, the adaptive controller must be robust, by construction, with respect to external and unknown disturbances. We focus mainly in the lateral dynamic of the plane, for this, an analysis of this dynamic is presented in section 2. Likewise, in this section we introduce the airframe addressed in this paper, the cross track error and the dynamic velocity of the plane with respect to the desired 
path. An adaptive control strategy is developed and presented in section 3 in order to follow the trajectory in presence of wind. Besides, the stability properties of the controller are discussed at the end of this section. The validation of the proposed control scheme is done in simulations and the main results are depicted in graphs in section 4. Additionally, an embedded control system was developed to validate the control algorithm, the main characteristics of this hardware platform are described in section 5. And finally in section 6 , the conclusion and future work are discussed.

\section{Aircraft system}

The dynamic characteristics of an airplane strongly depend on many parameters such as altitude, speed, configuration or environmental disturbances. As a result, its complete dynamic is nonlinear, uncertain and complex for control purposes. In this section we first introduce the airframe employed, then the lateral dynamics of the airplane in a non-steady atmosphere is derived.

A robust airframe possessing reliable flight characteristics is essential for real flight tests. Long duration flight and sufficient payload capacity to carry the weight of sensors and batteries are two features of great interest. Fig. 1 shows the commercially available Multiplex Twinstar II model used in our study. Its configuration is based on the classic aerodynamic layout and it is made of molded Elapor foam. Two brushless motors were mounted on the airfoil-shaped wings to power the airplane. A couple of ailerons, an elevator and a rudder are used as control surfaces and are actuated by servo motors. The technical characteristics of the Multiplex Twinstar II are given in Table 1. A payload of approximately $300 \mathrm{~g}$, consisting of sensors and a central processing unit, was added to the airframe as the embedded electronics. The developed hardware platform is described in detail in section 5 .

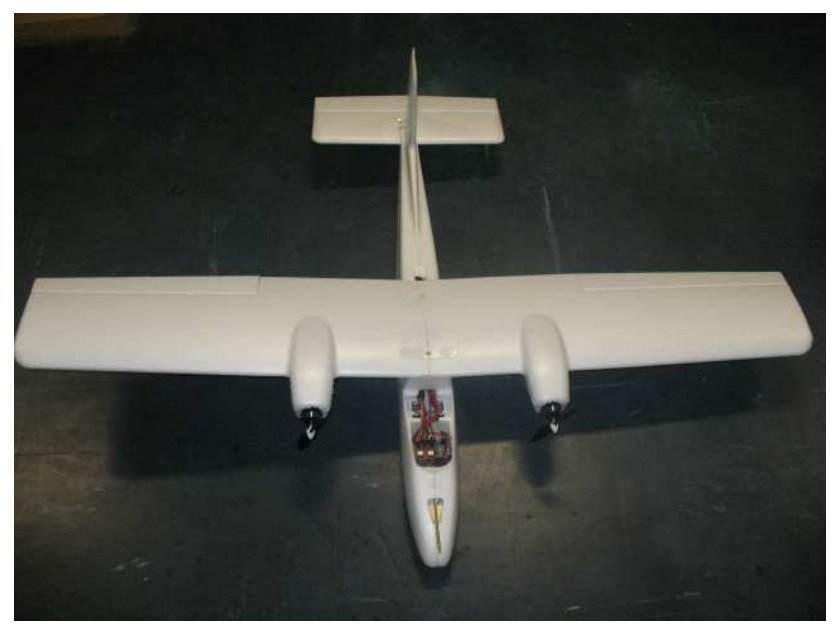

Fig. 1 Airplane model 
Table 1 Parameters of the airplane.

\begin{tabular}{ll}
\hline Parameter & Value \\
\hline Wingspan & $1420 \mathrm{~mm} / 55.9 \mathrm{in}$ \\
\hline Fuselage length & $1085 \mathrm{~mm} / 42.7 \mathrm{in}$ \\
\hline Wing area & $43 \mathrm{dm}^{2} / 666.5 \mathrm{inch}^{2}$ \\
\hline Weight approx. & $1340 \mathrm{~g} / 47.3 \mathrm{oz}$ \\
\hline Wing loading & $31.2 \mathrm{~g} / \mathrm{dm}^{2} / 10.3 \mathrm{oz} / \mathrm{sq} . \mathrm{ft}$ \\
\hline RC functions & Aileron, elevator, rudder, throttle \\
\hline
\end{tabular}

\subsection{Airplane dynamics}

The problem of trajectory following becomes complex when considering the complete dynamics of the airplane. In order to simplify the analysis and to better state the problem, let us explore only the airplane lateral motion and to consider that the path to be followed is a straight-line, as shown in Fig. 2. In addition, we assume that the airplane has a control system to hold the longitudinal variables stabilized to fly in level flight. That implies constant velocity, small roll and pitch angles and zero flight path angle, $\gamma$, see [13]. Consequently, the airplane velocity and the roll and pitch angles vary slowly compared to the other parameters and their time derivatives can be neglected in the flight dynamics. Under the above assumptions, the control problem to be solved simplifies to producing the yawing moment required for an airplane to change its direction according to the desired trajectory.

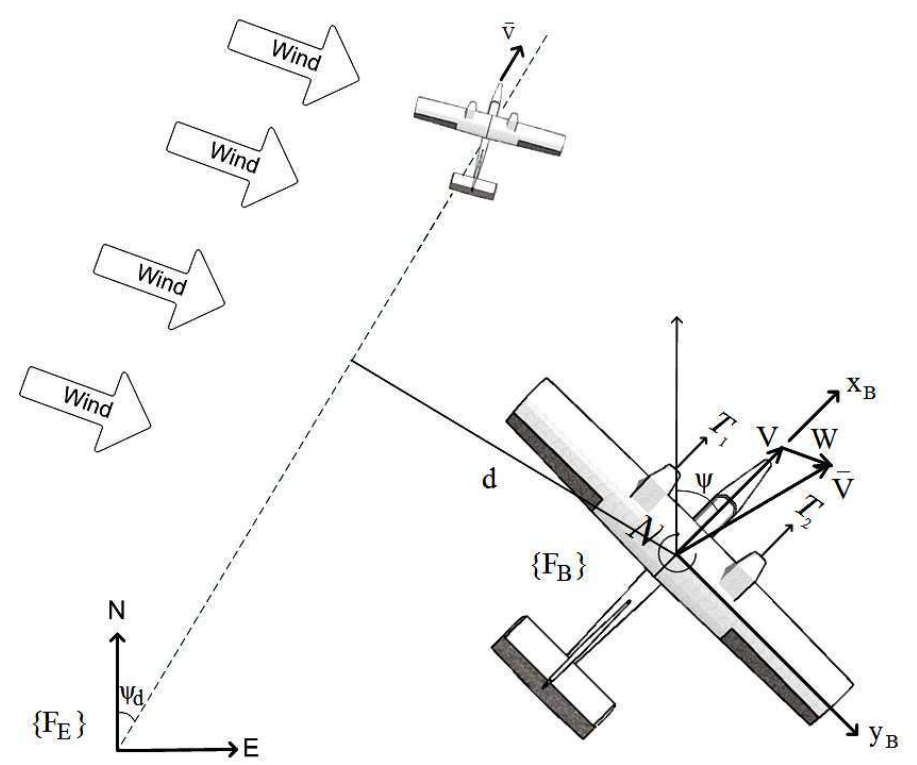

Fig. 2 Problem formulation for path following 
Two reference frames are used in order to derive the equations of motion: the Earth-fixed frame, denoted by $F_{E}$, and the body-fixed frame represented by $F_{B}$. $F_{E}$ and $F_{B}$ have two dimensions since only the lateral dynamics are considered in this study. The origin of $F_{B}$ coincides with the vehicle's center of mass and the direction of its axes is according to Fig. 2. $F_{E}$ is employed as an inertial frame and one of its axis is chosen northwards while the other points East.

In real conditions, the performance of an airplane is modified by environmental disturbances like wind. In such conditions it begins to sideslip or to be yawed out of its flight path. The classical relation of the aircraft velocity relative to the Earth is

$$
\bar{V}=V+W
$$

where $V=\left[\begin{array}{ll}u & v\end{array}\right]^{T}$ denotes the velocity of the aircraft relative to the local atmosphere and $W$ represents the wind relative to $F_{E}$. Besides, we only consider in this study the case of a crosswind which is defined as a lateral wind perpendicular to the vehicle but parallel to the ground, having North, $W_{N}$, and East velocity components, $W_{E}$.

Denote $\bar{V}_{B}=\left[\begin{array}{ll}u^{E} & v^{E}\end{array}\right]^{T}$ as the velocity of the aircraft relative to the Earth in the directions of the body frame axes. Thus, from (1) it follows

$$
\left[\begin{array}{l}
u^{E} \\
v^{E}
\end{array}\right]=\left[\begin{array}{l}
u \\
v
\end{array}\right]+B_{B}\left[\begin{array}{l}
W_{N} \\
W_{E}
\end{array}\right]
$$

where $B_{B}$ defines the complete transformation from $F_{E}$ to $F_{B}$ assuming constant pitch angle and it is given by

$$
B_{B}=\left(\begin{array}{cc}
c_{\theta} c_{\psi} & c_{\theta} s_{\psi} \\
s_{\phi} s_{\theta} c_{\psi}-c_{\phi} s_{\psi} & s_{\phi} s_{\theta} s_{\psi}+c_{\phi} c_{\psi}
\end{array}\right)
$$

where $s_{\theta}$ and $c_{\theta}$ denote $\sin (\theta)$ and $\cos (\theta)$, respectively.

Then, the differential equations for the coordinates of the flight path in $F_{E}$ are

$$
\left[\begin{array}{c}
\dot{x} \\
\dot{y}
\end{array}\right]=B_{B}^{T} \bar{V}_{B}
$$

or

$$
\begin{aligned}
& \dot{x}=u^{E} c_{\theta} c_{\psi}+v^{E} s_{\phi} s_{\theta} c_{\psi}-v^{E} c_{\phi} s_{\psi} \\
& \dot{y}=u^{E} c_{\theta} s_{\psi}+v^{E} s_{\phi} s_{\theta} s_{\psi}+v^{E} c_{\phi} c_{\psi}
\end{aligned}
$$

with

$$
\begin{aligned}
& u^{E}=u+W_{N} c_{\theta} c_{\psi}+W_{E} c_{\theta} s_{\psi} \\
& v^{E}=v+W_{N} s_{\phi} s_{\theta} c_{\psi}-W_{N} c_{\phi} s_{\psi}+W_{E} s_{\phi} s_{\theta} s_{\psi}+W_{E} c_{\phi} c_{\psi}
\end{aligned}
$$

where $x$ and $y$ represent the inertial position in the $x$-axis (North) and in the $y$-axis (East).

Remember that the pitch and roll angles are small so that $\sin \{\theta, \phi\} \approx 0$ and $\cos \{\theta, \phi\} \approx 1$. Moreover, considering a symmetrical airplane with a rigid spinning rotor placed in the front of its body, it can then be considered, without loss of 
generality, $V$ acting only in the $x$-axis, see Fig. 2 . Hence, the following expression can be stated

$$
\begin{aligned}
& v<<1 \\
& u \approx V
\end{aligned}
$$

and consequently

$$
\begin{aligned}
& \dot{x}=V \cos \psi+\omega \cos \psi_{\omega} \\
& \dot{y}=V \sin \psi+\omega \sin \psi_{\omega}
\end{aligned}
$$

where $\omega \cos \psi_{\omega}=W_{N}, \omega \sin \psi_{\omega}=W_{E}, \omega$ is the wind velocity and $\psi_{\omega}$ describes the wind direction. The motion of the airplane with respect to a stationary desired straight-line path of angle $\psi_{d}$ can then be expressed as

$$
\begin{aligned}
& \dot{x}=V \cos \left(\psi-\psi_{d}\right)+\omega \cos \left(\psi_{\omega}-\psi_{d}\right) \\
& \dot{y}=V \sin \left(\psi-\psi_{d}\right)+\omega \sin \left(\psi_{\omega}-\psi_{d}\right)
\end{aligned}
$$

Notice that the above equations are relatively proportional to the variation of the yaw angle. Considering that the motors of the airplane produce the same amount of thrust, then the yaw angular acceleration can be controlled using the rudder deflection. The differential equations describing this dynamics are

$$
\begin{aligned}
& \dot{\psi} \approx r \\
& \dot{r} \approx c \tau_{\psi}
\end{aligned}
$$

where $r$ stands for yaw rate, $\tau_{\psi}$ represents the yawing moment and $c$ is a constant related to the aircraft moment of inertia.

Fig. 3 shows an analysis of the nonlinear model of the airplane when it flies in stable or moving atmosphere. The desired trajectory is plotted in thick dashed line while the solid path describes the real airplane trajectory. The crosswind has North and East velocity components of $W_{N}=-3 \mathrm{~m} / \mathrm{s}$ and $W_{E}=5 \mathrm{~m} / \mathrm{s}$, respectively. The airplane velocity relative to the surrounding air mass is $20 \mathrm{~m} / \mathrm{s}$.

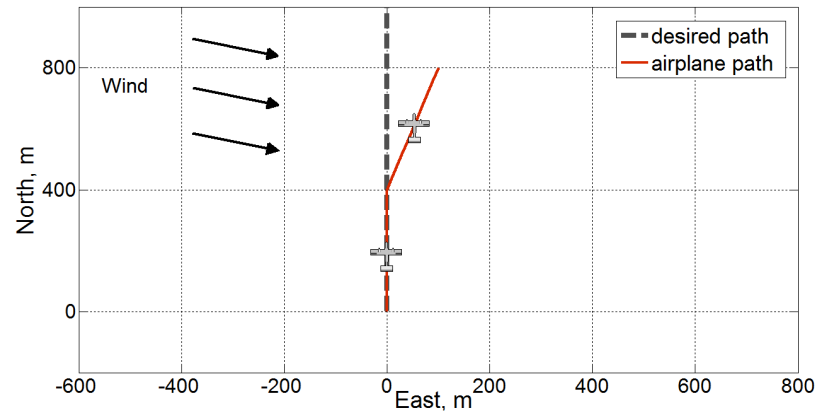

Fig. 3 Earth-Relative Aircraft Location. First, the plane flies in stable atmosphere and it is capable to follow the desired path. When the atmosphere moves relative to the Earth, the airplane diverge from the path. 


\section{Control design}

The main control objective is to obtain directional control in order to follow a desired trajectory even in presence of unknown crosswind. To simplify the analysis, let assume that the desired trajectory is aligned with the North axis of the reference frame, then, the desired path angle, $\psi_{d}$, is equal to zero. Therefore, the amount of the trajectory deviation will depend on the velocity of the airplane and wind and also on the angle of the wind in relation to the airplane. In addition we consider, for control design, that the wind velocity changes slowly such that it can be considered quasi-constant. However, it will be proved in simulations that the closed-loop system remains stable even with no constant wind.

Thus, without loss of generality, the airplane dynamics for trajectory following purpose can be defined as

$$
\begin{aligned}
\dot{d} \equiv \dot{y} & =V \sin \psi+k_{\omega} \\
\dot{\psi} & =r \\
\dot{r} & =c \tau_{\psi}
\end{aligned}
$$

where $k_{\omega}=\omega \sin \left(\psi_{\omega}\right)$ is considered, for control design, quasi-constant and it is due to the wind perturbation, and $d$ represents the cross track error from the desired trajectory.

To stabilize the system (2)-(4), the control law will be constructed using the adaptive backstepping approach. Define the following error variable

$$
e_{1}=d-d_{\min }
$$

where $d_{\text {min }}$ is the minimum constant distance from the desired trajectory. Thus,

$$
\dot{e}_{1}=V \sin \psi+k_{\omega}
$$

\subsection{Convergence of $e_{1}$ to zero}

Propose the following positive function

$$
V_{L_{1}}=\frac{1}{2} e_{1}^{2}
$$

thus

$$
\dot{V}_{L_{1}}=e_{1}\left(V \sin \psi+k_{\omega}\right)
$$

To stabilize $e_{1}$ we introduce $\psi^{v}$ as a virtual control in the following form

$$
V \sin \psi^{v}=-c_{1} e_{1}-\hat{k}_{\omega_{1}}
$$

where $\hat{k}_{\omega_{1}}$ is the estimate of $k_{\omega}$ and $c_{1}>0$ is a constant. Evaluating $\dot{V}_{L_{1}}$ when $\psi \rightarrow \psi^{v}$ it follows that

$$
\left.\dot{V}_{L_{1}}\right|_{\psi=\psi^{v}}=-c_{1} e_{1}^{2}+e_{1} \tilde{k}_{\omega_{1}}
$$

where $\tilde{k}_{\omega_{1}}=k_{\omega}-\hat{k}_{\omega_{1}}$. Notice from the above equation that if $\hat{k}_{\omega_{1}} \rightarrow k_{\omega}$ then $\dot{V}_{L_{1}} \leq 0$. Thus, rewriting $V_{L_{1}}$, it yields

$$
V_{L_{1}}=\frac{1}{2}\left(e_{1}^{2}+\frac{1}{\gamma_{1}} \tilde{k}_{\omega_{1}}^{2}\right)
$$


where $\gamma_{1}>0$ denotes a constant adaptation gain. Then

$$
\left.\dot{V}_{L_{1}}\right|_{\psi=\psi^{v}}=-c_{1} e_{1}^{2}+\left(e_{1}-\frac{\dot{\hat{k}}_{\omega_{1}}}{\gamma_{1}}\right) \tilde{k}_{\omega_{1}}
$$

Choosing the update law as

$$
\dot{\hat{k}}_{\omega_{1}}=\gamma_{1} e_{1}
$$

It follows that

$$
\left.\dot{V}_{L_{1}}\right|_{\psi=\psi^{v}}=-c_{1} e_{1}^{2}
$$

3.2 Convergence of $\psi$ to $\psi^{v}$

Define the error

$$
e_{2}=V \sin \psi-V \sin \psi^{v}=V \sin \psi+c_{1} e_{1}+\hat{k}_{\omega_{1}}
$$

and rewrite (6) in terms of $e_{1}$ and $e_{2}$

$$
\dot{e}_{1}=e_{2}-c_{1} e_{1}+\tilde{k}_{\omega_{1}}
$$

This implies that

$$
\dot{e}_{2}=V r \cos \psi+\left(\gamma_{1}-c_{1}^{2}\right) e_{1}+c_{1} e_{2}+c_{1} \tilde{k}_{\omega_{1}}
$$

Notice that $\cos \psi=\sqrt{1-(\sin \psi)^{2}}$. From (8)

$$
\sin \psi=\frac{e_{2}-c_{1} e_{1}-\hat{k}_{\omega_{1}}}{V}
$$

and assuming that $-\frac{\pi}{2}<\psi<\frac{\pi}{2}$ it follows that (10) becomes

$$
\dot{e}_{2}=r R+\left(\gamma_{1}-c_{1}^{2}\right) e_{1}+c_{1} e_{2}+c_{1} \tilde{k}_{\omega_{1}}
$$

with $R=\sqrt{V^{2}-\left(e_{2}-c_{1} e_{1}-\hat{k}_{\omega_{1}}\right)^{2}}$.

Introduce the following positive function

$$
V_{L_{2}}=V_{L_{1}}+\frac{1}{2} e_{2}^{2}=\frac{1}{2}\left(e_{1}^{2}+\frac{1}{\gamma_{1}} \tilde{k}_{\omega_{1}}^{2}+e_{2}^{2}\right)
$$

From (7), (9) and (11) the derivative reads

$$
\dot{V}_{L_{2}}=-c_{1} e_{1}^{2}+e_{2}\left[c_{1} e_{2}+e_{1}\left(\gamma_{1}+1-c_{1}^{2}\right)+c_{1} \tilde{k}_{\omega_{1}}+r R\right]
$$

By selecting the virtual control as

$$
r^{v} R=-e_{2}\left(c_{1}+c_{2}\right)-e_{1}\left(\gamma_{1}+1-c_{1}^{2}\right)-c_{1}\left(\hat{k}_{\omega_{2}}+\hat{k}_{\omega_{1}}\right)
$$


$\dot{V}_{L_{2}}$ becomes when $r \rightarrow r^{v}$

$$
\left.\dot{V}_{L_{2}}\right|_{r=r^{v}}=-c_{1} e_{1}^{2}-c_{2} e_{2}^{2}+c_{1} e_{2} \tilde{k}_{\omega_{2}}
$$

where $\tilde{k}_{\omega_{2}}=k_{\omega}-\hat{k}_{\omega_{2}}, \hat{k}_{\omega_{2}}$ represents a new estimate for $k_{\omega}$ and $c_{2}$ denotes a positive constant gain. Notice that if we had employed the existing estimate $\hat{k}_{\omega_{1}}$, we would have had no design freedom left to cancel the unknown parameter from $\dot{V}_{L_{2}}$. Additionally, $\hat{k}_{\omega_{2}}$ could be seen as a factor correction for $\hat{k}_{\omega_{1}}$.

Notice from the above equation that if $\hat{k}_{\omega_{2}} \rightarrow k_{\omega}$ then $\dot{V}_{L_{2}} \leq 0$. Thus, rewriting $V_{L_{2}}$, it yields

$$
V_{L_{2}}=V_{L_{1}}+\frac{1}{2}\left(e_{2}^{2}+\frac{1}{\gamma_{2}} \tilde{k}_{\omega_{2}}^{2}\right)
$$

with $\gamma_{2}>0$ and constant. Hence $\dot{V}_{L_{2}}$ becomes

$$
\left.\dot{V}_{L_{2}}\right|_{r=r^{v}}=c_{1} e_{1}^{2}-c_{2} e_{2}^{2}+\tilde{k}_{\omega_{2}}\left(c_{1} e_{2}-\frac{\dot{\hat{k}}_{\omega_{2}}}{\gamma_{2}}\right)
$$

Proposing the update law

$$
\dot{\hat{k}}_{\omega_{2}}=\gamma_{2} c_{1} e_{2}
$$

then, it follows

$$
\left.\dot{V}_{L_{2}}\right|_{r=r^{v}}=c_{1} e_{1}^{2}-c_{2} e_{2}^{2}
$$

\subsection{Convergence of $r$ to $r^{v}$}

Let us define the third error variable

$$
\begin{aligned}
e_{3} & =r R-r^{v} R \\
& =r R+L_{2} e_{2}+L_{1} e_{1}+c_{1}\left(\hat{k}_{\omega_{2}}-\hat{k}_{\omega_{1}}\right)
\end{aligned}
$$

where $L_{1}=1-c_{1}^{2}+\gamma_{1}, L_{2}=c_{1}+c_{2}$. Rewriting the error system representation, we obtain

$$
\left[\begin{array}{c}
\dot{e}_{1} \\
\dot{e}_{2}
\end{array}\right]=\left[\begin{array}{cc}
-c_{1} & 1 \\
-1 & -c_{2}
\end{array}\right]\left[\begin{array}{l}
e_{1} \\
e_{2}
\end{array}\right]+\left[\begin{array}{c}
\tilde{k}_{\omega_{1}} \\
e_{3}+c_{1} \tilde{k}_{\omega_{2}}
\end{array}\right]
$$

thus, the derivative of $e_{3}$ yields

$$
\begin{aligned}
\dot{e}_{3}= & c \tau_{\psi} R-\frac{r\left(e_{2}-c_{1} e_{1}-\hat{k}_{\omega_{1}}\right)\left(e_{3}-L_{2} e_{2}-L_{1} e_{1}+c_{1} \hat{k}_{\omega_{1}}-c_{1} \hat{k}_{\omega_{2}}\right)}{R} \\
& +L_{2} e_{3}+L_{3} e_{2}+L_{4} e_{1}+c_{1} L_{2} \tilde{k}_{\omega_{2}}+L_{1} \tilde{k}_{\omega_{1}}
\end{aligned}
$$

with $L_{3}=-c_{1} c_{2}-c_{1}^{2}-c_{2}^{2}+1+\gamma_{1}+c_{1}^{2} \gamma_{2}$ and $L_{4}=-2 c_{1}-c_{2}+c_{1}^{3}-2 c_{1} \gamma_{1}$.

Finally, introduce the following Lyapunov function

$$
V_{L}=\frac{1}{2}\left(e_{1}^{2}+\frac{1}{\gamma_{1}} \tilde{k}_{\omega_{1}}^{2}+e_{2}^{2}+\frac{1}{\gamma_{2}} \tilde{k}_{\omega_{2}}^{2}+e_{3}^{2}\right)
$$

then

$$
\dot{V}_{L}=-c_{1} e_{1}^{2}-c_{2} e_{2}^{2}+e_{3}\left(\dot{e}_{3}+e_{2}\right)
$$


Propose the control input as

$$
\begin{aligned}
c \tau_{\psi}= & -\frac{e_{3}\left(L_{2}+c_{3}\right)+e_{2}\left(L_{3}+1-r^{2}\right)+e_{1}\left(L_{4}+c_{1} r^{2}\right)}{R} \\
& -\frac{\hat{k}_{\omega_{3}}\left(L_{1}+c_{1} L_{2}\right)-\hat{k}_{\omega_{2}} c_{1} L_{2}-\hat{k}_{\omega_{1}}\left(L_{1}-r^{2}\right)}{R}
\end{aligned}
$$

where $\tilde{k}_{\omega_{3}}=k_{\omega}-\hat{k}_{\omega_{3}}$ and $c_{3}$ is a positive constant gain. Notice that the unknown term $k_{\omega}$ appears again in $\dot{V}_{L}$, thus we propose a correction factor in order to realize the convergence of the states.

Introducing the above into (13), we have

$$
\dot{V}_{L}=-c_{1} e_{1}^{2}-c_{2} e_{2}^{2}-c_{3} e_{3}^{2}+e_{3}\left(L_{1}+c_{1} L_{2}\right) \tilde{k}_{\omega_{3}}
$$

Observe that $\dot{V}_{L} \leq 0$ if $\hat{k}_{\omega_{3}} \rightarrow k_{\omega}$. Therefore augmenting $V_{L}$, it yields

$$
V_{L}=\frac{1}{2}\left(e_{1}^{2}+\frac{1}{\gamma_{1}} \tilde{k}_{\omega_{1}}^{2}+e_{2}^{2}+\frac{1}{\gamma_{2}} \tilde{k}_{\omega_{2}}^{2}+e_{3}^{2}+\frac{1}{\gamma_{3}} \tilde{k}_{\omega_{3}}^{2}\right)
$$

and

$$
\dot{V}_{L}=-c_{1} e_{1}^{2}-c_{2} e_{2}^{2}-c_{3} e_{3}^{2}+\tilde{k}_{\omega_{3}}\left[e_{3}\left(L_{1}+c_{1} L_{2}\right)-\frac{\dot{\hat{k}}_{\omega_{3}}}{\gamma_{3}}\right]
$$

Choosing

$$
\dot{\hat{k}}_{\omega_{3}}=\gamma_{3}\left(L_{1}+c_{1} L_{2}\right) e_{3}
$$

$\dot{V}_{L}$ becomes

$$
\dot{V}_{L}=-c_{1} e_{1}^{2}-c_{2} e_{2}^{2}-c_{3} e_{3}^{2}
$$

The error representation of the closed-loop adaptive system is summarized below

$$
\begin{gathered}
{\left[\begin{array}{c}
\dot{e}_{1} \\
\dot{e}_{2} \\
\dot{e}_{3}
\end{array}\right]=\left[\begin{array}{ccc}
-c_{1} & 1 & 0 \\
-1 & -c_{2} & 1 \\
0 & -1 & -c_{3}
\end{array}\right]\left[\begin{array}{l}
e_{1} \\
e_{2} \\
e_{3}
\end{array}\right]+\left[\begin{array}{c}
\tilde{k}_{\omega_{1}} \\
c_{1} \tilde{k}_{\omega_{2}} \\
L_{5} \tilde{k}_{\omega_{3}}
\end{array}\right]} \\
{\left[\begin{array}{l}
\dot{\hat{k}}_{\omega_{1}} \\
\dot{\hat{k}}_{\omega_{2}} \\
\dot{\hat{k}}_{\omega_{3}}
\end{array}\right]=\left[\begin{array}{ccc}
\gamma_{1} & 0 & 0 \\
0 & c_{1} \gamma_{2} & 0 \\
0 & 0 & L_{5} \gamma_{3}
\end{array}\right]\left[\begin{array}{l}
e_{1} \\
e_{2} \\
e_{3}
\end{array}\right]}
\end{gathered}
$$

where $L_{5}=c_{1} c_{2}+\gamma_{1}+1$.

Rewriting the control input $c \tau_{\psi}$ in terms of $d, \psi, r$ we have

$$
c \tau_{\psi}=\tan \psi\left(r^{2}-L_{6}\right)-L_{7} r-\frac{L_{8} d+L_{9} \hat{k}_{\omega_{1}}+L_{10} \hat{k}_{\omega_{2}}+L_{11} \hat{k}_{\omega_{3}}}{V \cos \psi}
$$

with the updated parameters

$$
\begin{aligned}
& \dot{\hat{k}}_{\omega_{1}}=\gamma_{1} d \\
& \dot{\hat{k}}_{\omega_{2}}=\gamma_{2} c_{1}\left(V \sin \psi+c_{1} d+\hat{k}_{\omega_{1}}\right) \\
& \dot{\hat{k}}_{\omega_{3}}=\gamma_{3} L_{11} V\left[r \cos \psi+L_{2} \sin \psi\right]+\gamma_{3} L_{11}\left[d L_{11}+c_{1} \hat{k}_{\omega_{2}}+c_{2} \hat{k}_{\omega_{1}}\right]
\end{aligned}
$$


where

$$
\begin{aligned}
L_{6} & =1+L_{2} c_{3}+L_{2}^{2}+L_{3} \\
L_{7} & =L_{2}+c_{3} \\
L_{8} & =L_{7}\left(L_{1}+c_{1} L_{2}\right)+c_{1}\left(L_{3}+1\right)+L_{4} \\
L_{9} & =1-c_{1} L_{7}+L_{3}-L_{1}+L_{2} L_{7} \\
L_{10} & =c_{1} L_{7}-c_{1} L_{2} \\
L_{11} & =L_{1}+c_{1} L_{2}
\end{aligned}
$$

Notice from (14) that $\dot{V}_{L} \leq 0$ and it estates the global stability of the equilibrium $\left(e_{i}, \tilde{k}_{\omega_{i}}\right)=(0,0)$. From the LaSalle-Yoshizawa theorem, we have that $e_{i}$ and $\tilde{k}_{\omega_{i}} ; i=1,2,3$; are bounded and go to zero as $t \rightarrow \infty$. From (5) it follows that $d \rightarrow d_{\text {min }}$. (8) implies that $\hat{k}_{\omega_{1}}$ is also bounded and

$$
\lim _{t \rightarrow \infty} \psi=\arcsin \left(-\frac{\hat{k}_{\omega_{1}}}{V}\right)
$$

Observe that from (12) $r$ is bounded and $r \rightarrow 0$. On the other hand, from (16) it follows that $c \tau_{\psi}$ is bounded.

LaSalle's invariance principle assures that the state $\left(e_{i}, \tilde{k}_{\omega_{i}}\right)$ converges to the largest invariant set $M$ contained in $\left\{\left(e_{1}, e_{2}, e_{3}, \tilde{k}_{\omega_{1}}, \tilde{k}_{\omega_{2}}, \tilde{k}_{\omega_{3}}\right) \in \mathbb{R}^{6} \mid \dot{V}_{L}=0\right\}$. On this invariant set, we have $e_{i} \equiv 0$ and $\dot{e}_{i} \equiv 0$. From (15) it yields $\dot{\tilde{k}}_{\omega_{i}}=0$ and $\tilde{k}_{\omega_{i}}=0$. Thus, the largest invariant set $M$ is

$$
\begin{aligned}
M= & \left\{\left(e_{i}, \tilde{k}_{\omega_{i}}\right) \in \mathbb{R}^{6} \mid e_{i}=0, \tilde{k}_{\omega_{i}}=0\right\} \\
= & \left\{\left(d, \psi, r, \hat{k}_{\omega_{1}}, \hat{k}_{\omega_{2}}, \hat{k}_{\omega_{3}}\right) \in \mathbb{R}^{6} \mid\left(d, \psi, r, \hat{k}_{\omega_{1}}, \hat{k}_{\omega_{2}}, \hat{k}_{\omega_{3}}\right)\right. \\
& \left.=\left(0, \arcsin \left(-\frac{\hat{k}_{\omega_{1}}}{V}\right), 0, k_{\omega}, k_{\omega}, k_{\omega}\right)\right\}
\end{aligned}
$$

The manifold $M$ is the single point $d=0, \psi=\arcsin \left(-\frac{\hat{k}_{\omega_{1}}}{V}\right), r=0, \hat{k}_{\omega_{i}}=k_{\omega}$ for $i=1,2$ and 3 , which is globally asymptotically stable.

\section{Simulation results}

The proposed control strategy was validated in closed-loop system in simulations with various wind conditions. Remember that we have considered the desired trajectory aligned with the North axis of the inertial frame which makes the desired path angle $\psi_{d}=0^{\circ}$. In addition, the airplane is flying with a constant speed equal to $20 \mathrm{~m} / \mathrm{s}$ and the crosswind has a direction West-East perpendicular to the desired path. For a smoother convergence we have used the following parameters in simulations: $c_{1}=c_{3}=1.5 ; c_{2}=1.3 ; \gamma_{1}=1 ; \gamma_{2}=1.1 ; \gamma_{3}=1.4$. 


\subsection{Case constant wind}

Several simulations were performed to validate the controller and representative results are presented. The first simulations were carried out with a constant wind velocity of $7 \mathrm{~m} / \mathrm{s}$. The initial conditions are: $d=2 \mathrm{~m}, \psi=-10^{\circ}$ and $r=0 \mathrm{rad} / \mathrm{s}$. For comparative control purpose, a standard nonlinear backstepping algorithm was developed (see Appendix A) to control the system (2)-(4) and it is given by

$$
c \tau_{\psi_{b}}=-3 r+\tan \psi\left(r^{2}-5\right)-\frac{3 d+5 k_{\omega}}{V \cos \psi}
$$

In Fig. 4 we show the time evolution of the aircraft deviation from the desired trajectory for constant wind when employing the controllers (16) and (18). The wind parameter, denoted by $k_{\omega}$, is not known and therefore considered zero in (18). Notice from this figure that the controller proposed in (16) is able to provide cross track error regulation due to the adaptation laws presented in (15). For this case, the closed-loop adaptive system shows good response even in presence of unknown disturbance.

Fig. 5 reveals the fact that to maintain alignment with the desired trajectory during a crosswind flight requires the controller to fly the airplane at a sideslip angle. Indeed, when the position error converges to zero, the yaw angle is stabilized around a constant value and the airplane keeps moving toward North. Notice that the yaw angle is nonzero unless the atmosphere is at rest.

On the other hand, the proposed adaptation scheme guarantees the convergence of the unknown parameter estimates towards its true constant value, see Fig. 6. The Lyapunov function, plotted in Fig. 7, is semi-positive definite and continually decreasing which proves the stability properties of the system. Indeed, in Fig. 8 we illustrate the control input response.

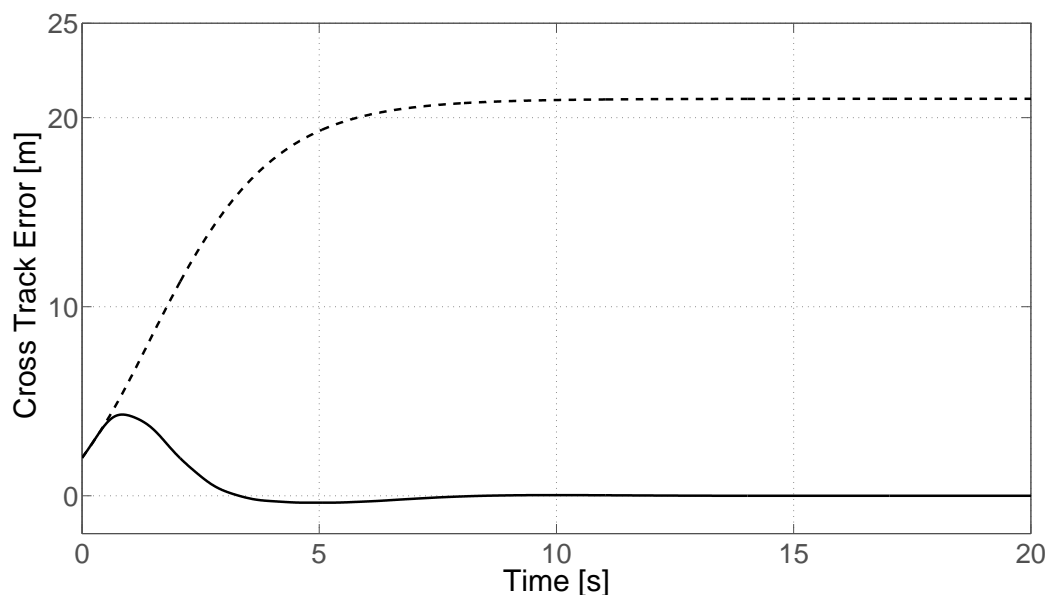

Fig. 4 Position error for unknown wind. Solid line represents the proposed controller (16) whilst dashed line the standard backstepping control algorithm (18). 


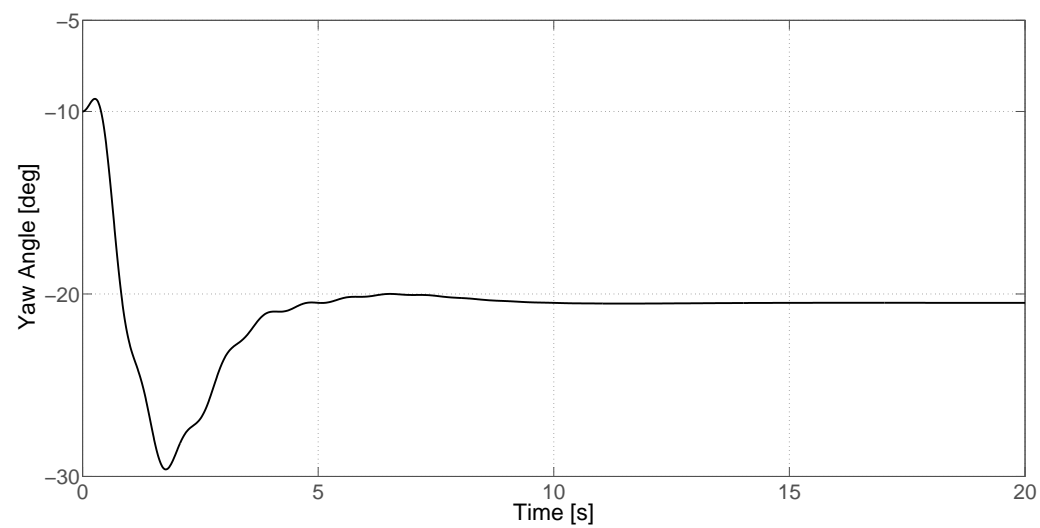

Fig. 5 Yaw angle for $k_{\omega}=7 \mathrm{~m} / \mathrm{s}$

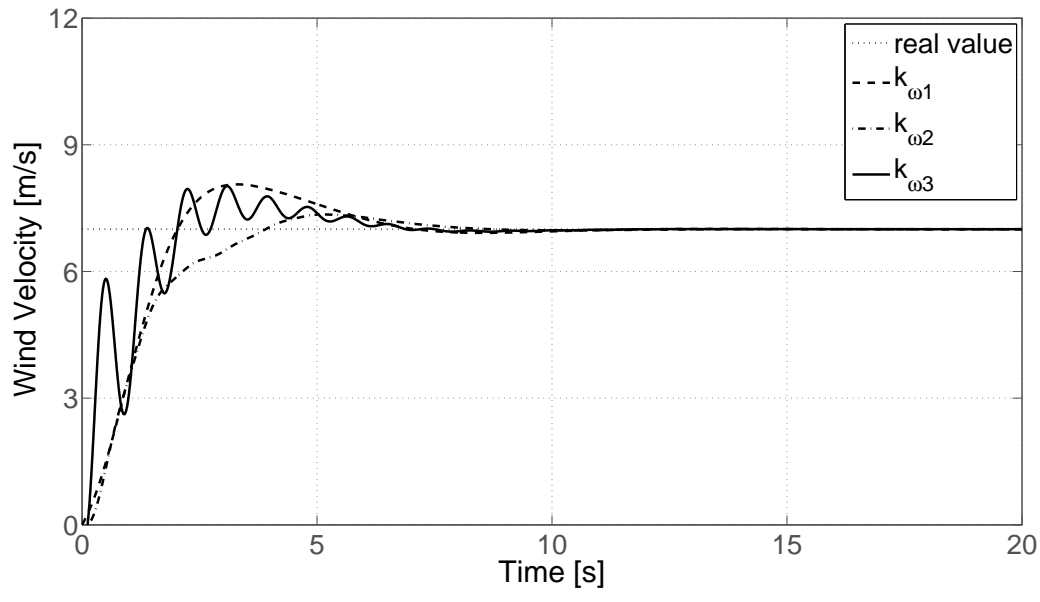

Fig. 6 Parameter estimation for $k_{\omega}=7 \mathrm{~m} / \mathrm{s}$

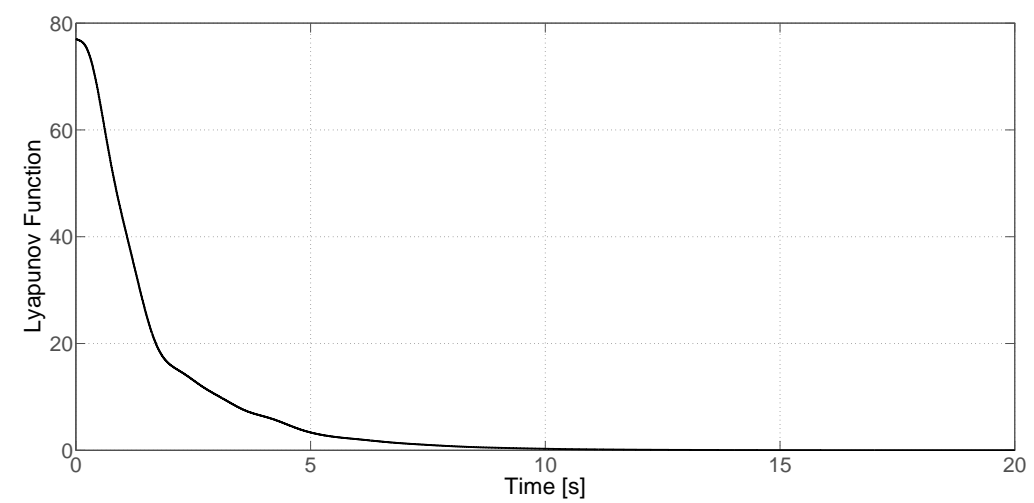

Fig. 7 Lyapunov function for $k_{\omega}=7 \mathrm{~m} / \mathrm{s}$ 


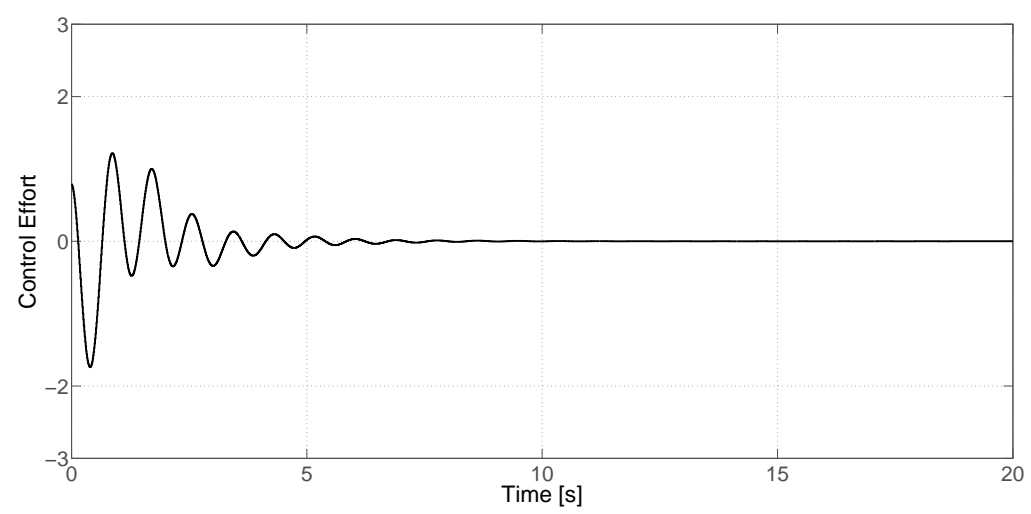

Fig. 8 Control input for $k_{\omega}=7 \mathrm{~m} / \mathrm{s}$

4.2 Case variable wind and sensor noise

In order to demonstrate the robustness (in simulation) of the proposed control algorithm, some variations are included in the wind parameters and noise is considered in sensor measurements. For this purpose, we assume that the wind varies in magnitude and orientation, as shown in Fig. 9. Notice in this figure that, at time 20s, a sudden increase of $2 \mathrm{~m} / \mathrm{s}$ is presented in speed of the wind.

The airspeed, the distance relative to the path, the yaw angle and the yaw rate are computed by sensors placed onboard whose measurements are affected by random variations. To approximate as much as possible a real life scenario, let us evaluate the controller in presence of normal gaussian noise. To this end, the outputs of the airspeed and distance sensors are perturbed by the noise represented in Fig. 10(a) while the outputs of the yaw angle yaw rate sensors by the noise represented in Fig. 10(b).
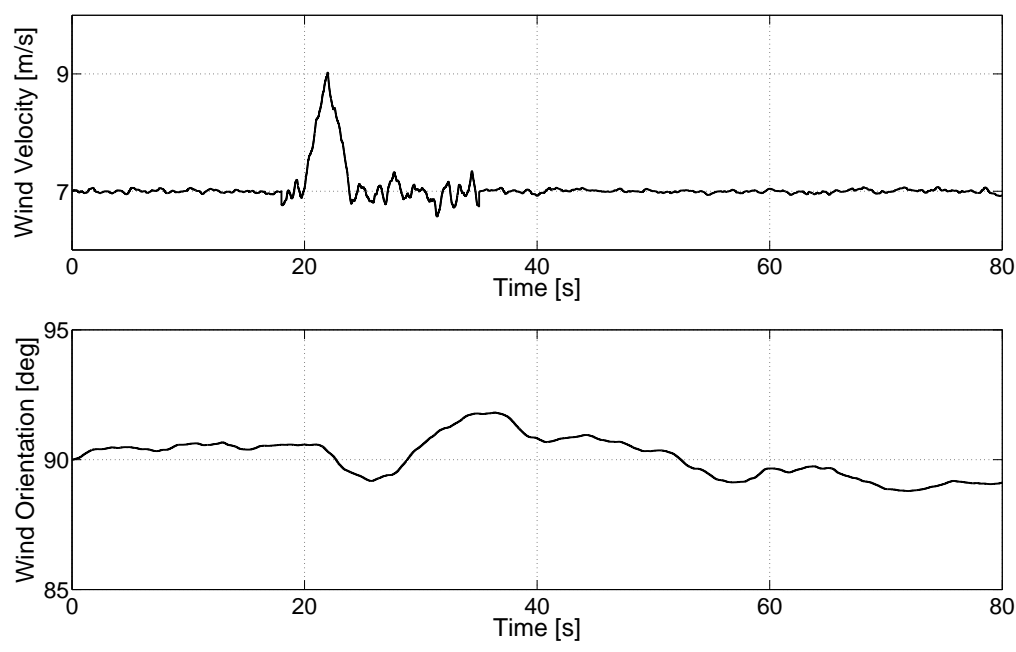

Fig. 9 Variable wind gust 


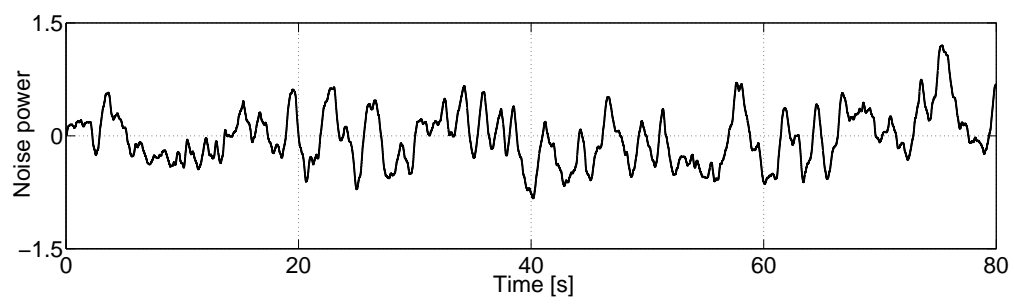

a. Noise of the airspeed and distance sensors

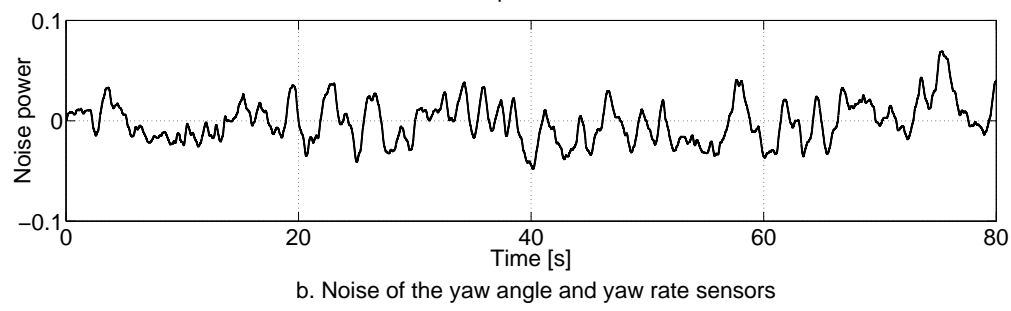

Fig. 10 Normal Gaussian noise

The overall system has been simulated for the case when the path to be followed is a four straight-line segments combination. The first segment is aligned with the reference while the angles of the three following segments are $55^{\circ}, 110^{\circ}$ and $160^{\circ}$ relative to the North axis, see Fig. 11. Initial deviation of the airplane from the path is $-15 \mathrm{~m}$ while its initial orientation is considered as for the previous simulations.

The main results are displayed in Figures 11 - 15. Observe that path following is achieved even in presence of sudden changes in path direction, variations in wind parameters and sensor noise. The wind deviates the airplane from the reference trajectory toward the wind direction but the controller (16) is able to recover the aircraft and to converge the position error to zero, see Fig. 12.

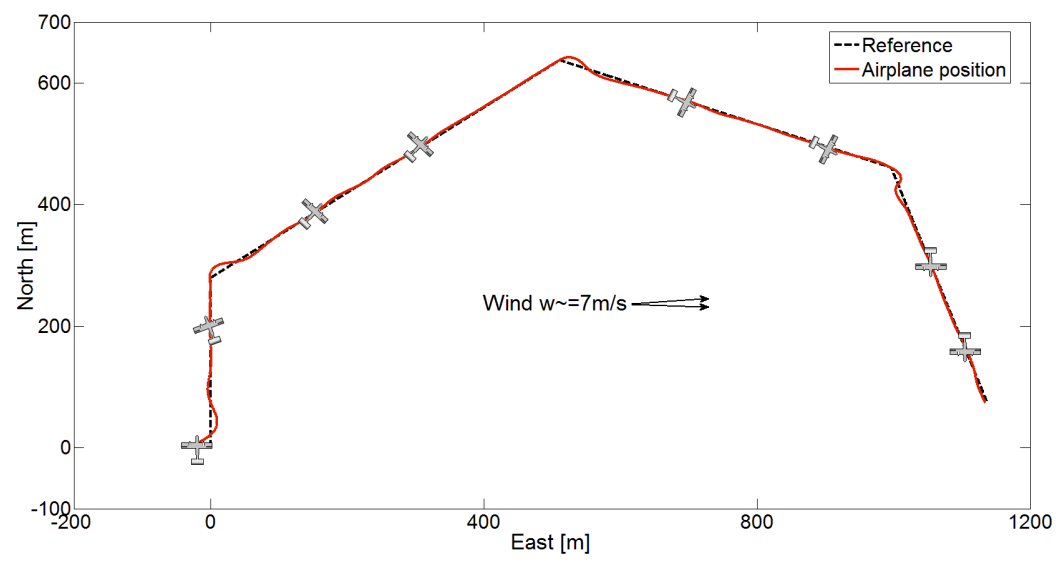

Fig. 11 The path to be followed consists of a combination of four straight-line segments. 
When aligned with the reference trajectory, the airplane is flown at a sideslip angle to maintain directional control, see Fig. 13. The adaptation laws are used to estimate the value of $k_{\omega}=\omega \sin \left(\psi_{\omega}-\psi_{d}\right)$, which varies mainly according to $\psi_{d}$ as shown in Fig. 14. Notice from this figure that the convergence time is relatively small and that the estimated unknown parameters are in agreement with the real value. The control effort is illustrated in Fig. 15.

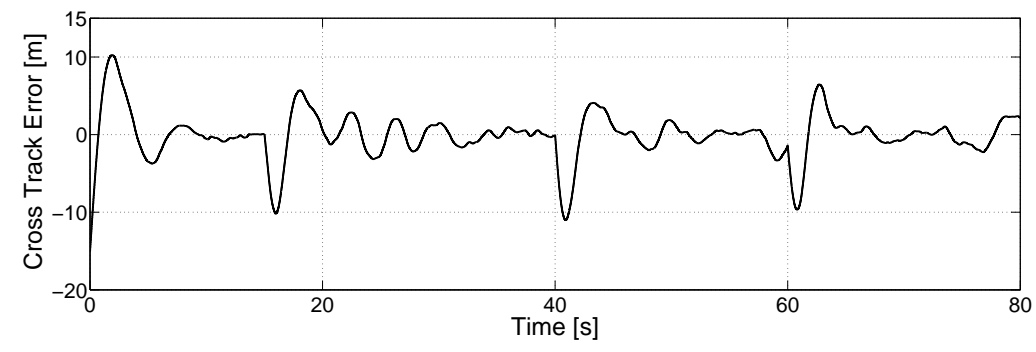

Fig. 12 Position error for variable wind and sensor noise

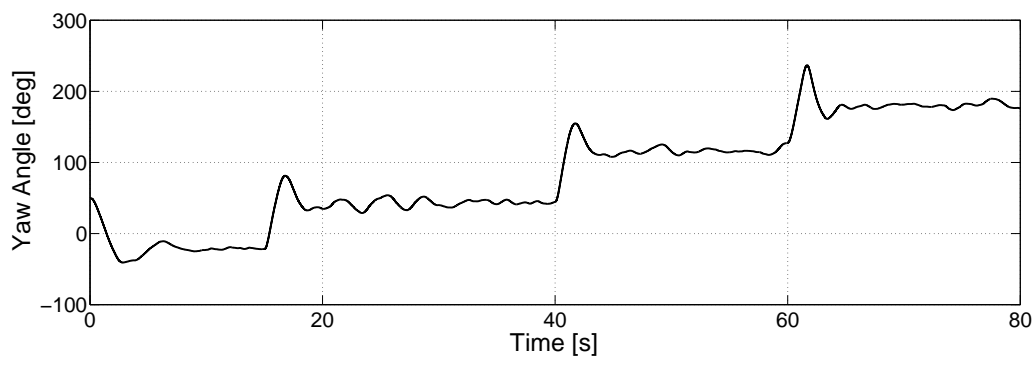

Fig. 13 Yaw angle for variable wind and sensor noise

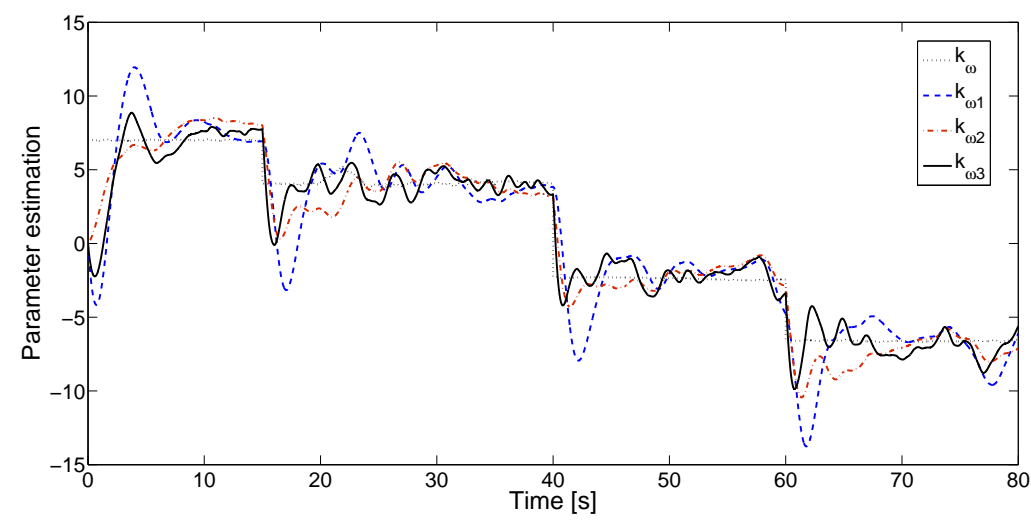

Fig. 14 Parameter estimation for variable wind and sensor noise 


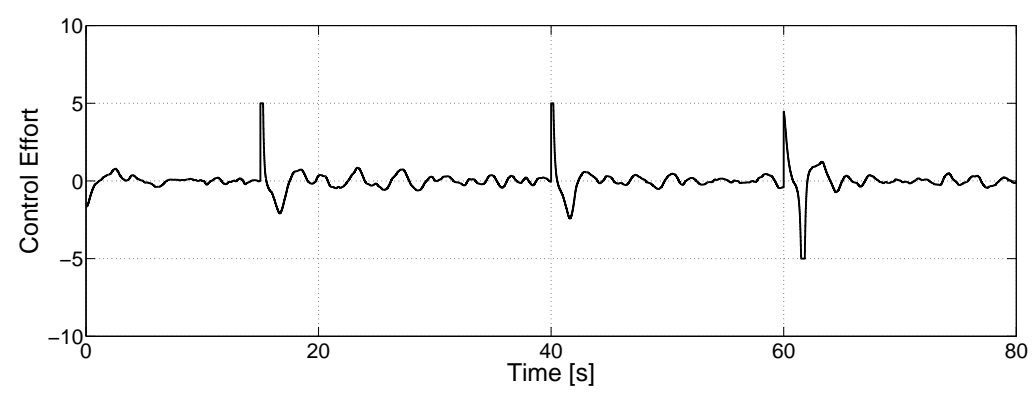

Fig. 15 Control input for variable wind and sensor noise

\section{Onboard electronics}

In this section we introduce an overview of the onboard hardware developed in order to carry out flight tests. The airplane used is the Multiplex TwinStar II. The central processing unit, represented by the RabbitCore RCM4300 Microprocessor, collects the measurements of the IMU (Inertial Measurement Unit employed to estimate the airplane attitude and angular rates), of the airspeed sensor and of the GPS system, to compute the control law. The control responses are sent to the servo signal generator/receiver unit and also to the two electric speed controllers to activate the brushless motors. Indeed, a modem is added to send and receive data from a base station. The electronic scheme is presented in Fig. 17.

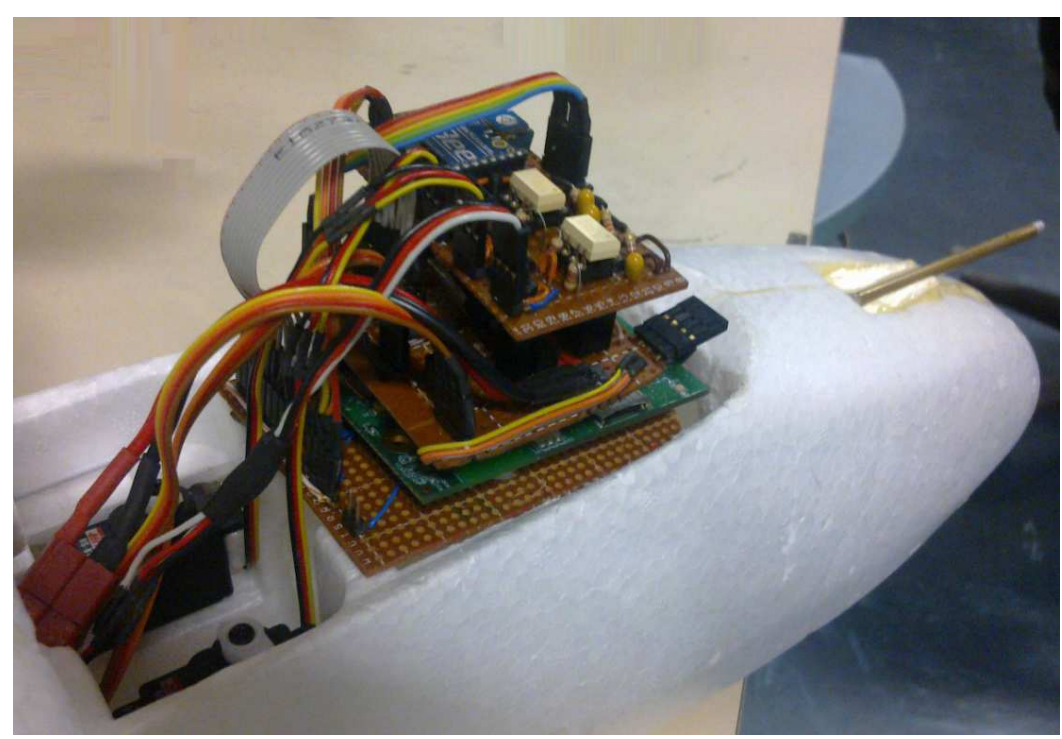

Fig. 16 The onboard electronics 


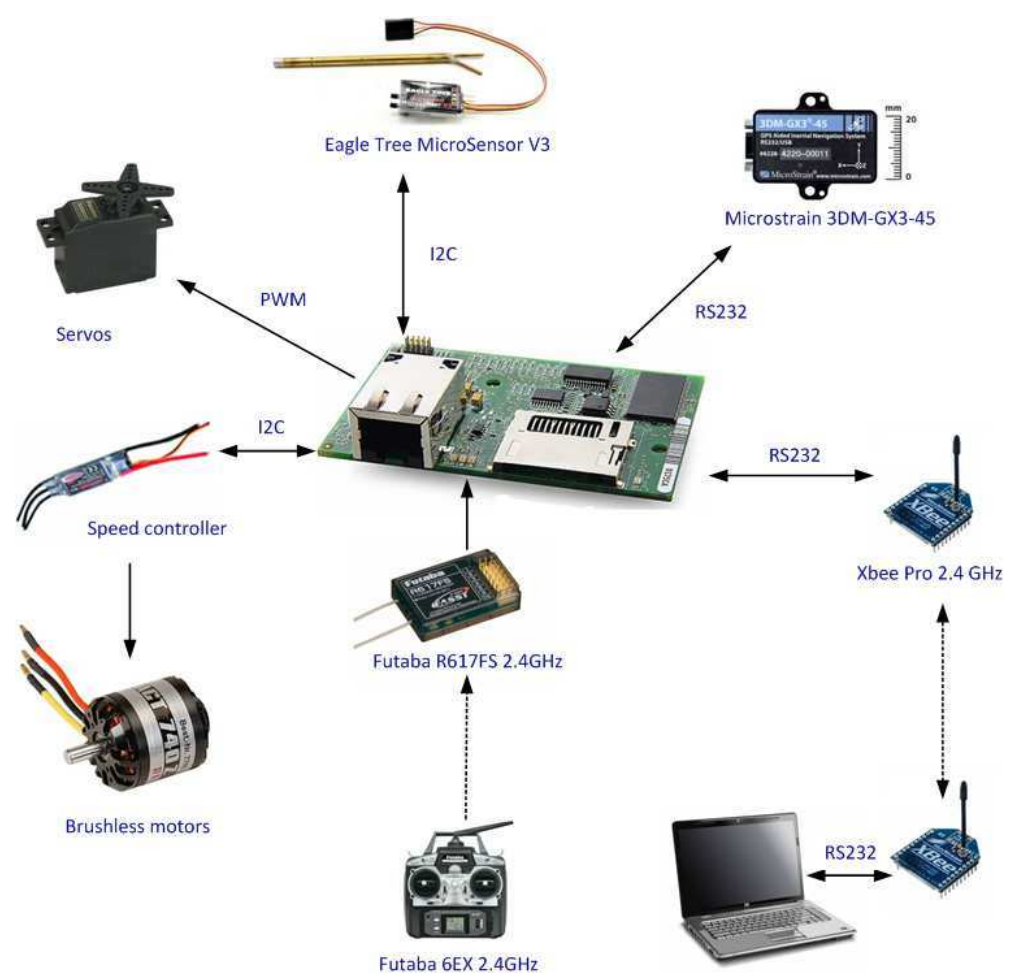

Fig. 17 The electronic scheme

\section{CONCLUSIONS AND FUTURE WORK}

An adaptive control algorithm based on the backstepping approach has been proposed in this paper. The control strategy was focused on reducing the position deviation of the airplane with respect to a desired path in the lateral dynamics in presence of unknown wind. The control scheme was derived considering adaptation laws to estimate the unknown wind parameters. The closed-loop system was evaluated in several simulations and the main results, showing the good performance, were introduced by some graphs. An embedded control system was developed in order to validate the control strategy in flight tests. Future work will include real time implementation of the flight controller using the developed hardware platform.

\section{References}

1. C. Silvestre, A. Pascoal and I. Kaminer, On the design of gain-scheduled tra jectory tracking controllers, International Journal of Robust and Nonlinear Control 12, 797-839, 2002.

2. W. J. Rugh and J. S. Shamma, Research on Gain Scheduling, Automatica 36, pg. 1401-1425, 2000 . 
3. J. S. Shamma and M. Athans, Guaranteed Properties of Gain Scheduled Control of Linear Parameter-Varying Plants, Automatica, Vol. 27, No. 3, pp. 559-565, May 1991.

4. T. M. Adami and J. Jim Zhu, 6DOF flight control of fixed-wing aircraft by Trajectory Linearization, Proceedings of the 2011 American Control Conference, pg. 1610-1617, June 2011.

5. D. R. Nelson, D. B. Barber, T. W. McLain and R. W. Beard, Vector field path following for small unmanned air vehicles, IEEE Transactions on Robotics and Automation 23(3), pp. 519-529, 2007.

6. J. J. E. Slotine and W. Li, Applied Nonlinear Control, Prentice Hall, Englewood Cliffs, 1991.

7. H.Khalil, Nonlinear Systems, Macmillan Publishing Company, New York, 1992.

8. P. V. Kokotovic, The joy of Feedback : Nonlinear and Adaptive, IEEE Control Systems, Vol. 12, No. 3, pp 7-17, 1992.

9. M. Krstic, I. Kanellakopoulos and P. V. Kokotovic, Nonlinear and Adaptive Control Design, John Wiley \& Sons, New York, 1995.

10. O. Harkergard, Backstepping and Control Allocation with Applications to Flight Control, Ph.D. thesis, Linkoping University, 2003.

11. L. Sonneveldt, Q.P. Chu and J.A. Mulder, Adaptive Backstepping Flight Control for Modern Fighter Aircraft, Advances in Flight Control Systems, Agneta Balint (Ed.), ISBN: 978953-307-218-0, InTech, 2011.

12. F. Gavilan, J. A. Acosta and R. Vazquez, Control of the longitudinal flight dynamics of an UAV using adaptive backstepping, IFAC World Congress, 2011.

13. Bernard Etkin, Dynamics of Atmospheric Flight, John Wiley \& Sons, New York, 1972.

14. http://www.stack.nl/ jwk/latex/ 


\section{A Standard nonlinear backstepping design}

Let us rewrite the nonlinear system described by (2)-(4)

$$
\begin{aligned}
\dot{d} & =V \sin \psi+k_{\omega} \\
\dot{\psi} & =r \\
\dot{r} & =c \tau_{\psi}
\end{aligned}
$$

where $k_{\omega}$ is a constant perturbation due to the wind. We intend to achieve regulation of $d(t)$ designing backstepping control, for this purpose we define the following error variable

$$
e_{1}=d-d_{\text {min }}
$$

where $d_{\min }$ is the minimum constant distance from the desired trajectory. The dynamics of $e_{1}$ yields

$$
\dot{e}_{1}=V \sin \psi+k_{\omega}
$$

Let us consider the following positive function

$$
V_{L_{1}}=\frac{1}{2} e_{1}^{2}
$$

thus

$$
\dot{V}_{L_{1}}=e_{1}\left(V \sin \psi+k_{\omega}\right)
$$

The $e_{1}$ term can be stabilized if we introduce $\psi^{v}$ as virtual control in the form

$$
V \sin \psi^{v}=-e_{1}-k_{\omega}
$$

Evaluating $\dot{V}_{L_{1}}$ when $\psi \rightarrow \psi^{v}$ it follows that

$$
\left.\dot{V}_{L_{1}}\right|_{\psi=\psi^{v}}=-e_{1}^{2}
$$

Since $\psi$ is not the real control, let us define the deviation from its desired value

$$
e_{2}=V \sin \psi-V \sin \psi^{v}=V \sin \psi+e_{1}+k_{\omega}
$$

and rewrite (19) in terms of $e_{1}$ and $e_{2}$

$$
\dot{e}_{1}=e_{2}-e_{1}
$$

This implies that

$$
\dot{e}_{2}=V r \cos \psi+e_{2}-e_{1}
$$

Notice that $\cos \psi=\sqrt{1-(\sin \psi)^{2}}$. From $(20)$

$$
\sin \psi=\frac{e_{2}-e_{1}-k_{\omega}}{V}
$$

and assuming that $-\frac{\pi}{2}<\psi<\frac{\pi}{2}$ it follows that (22) becomes

$$
\dot{e}_{2}=r R+e_{2}-e_{1}
$$

with $R=\sqrt{V^{2}-\left(e_{2}-e_{1}-k_{\omega}\right)^{2}}$. Let us consider the positive definite function

$$
V_{L_{2}}=\frac{1}{2}\left(e_{1}^{2}+e_{2}^{2}\right)
$$

whose derivative is

$$
\dot{V}_{L_{2}}=-e_{1}^{2}+e_{2}\left(e_{2}+r R\right)
$$

Using the virtual control $r^{v}$ in the form

$$
r^{v} R=-2 e_{2}
$$


$\dot{V}_{L_{2}}$ becomes when $r \rightarrow r^{v}$

$$
\left.\dot{V}_{L_{2}}\right|_{r=r^{v}}=-e_{1}^{2}-e_{2}^{2}
$$

Let $e_{3}$ be the deviation of $r$ from its desired value

$$
e_{3}=r R-r^{v} R=r R+2 e_{2}
$$

This implies

$$
r=\frac{e_{3}-2 e_{2}}{R}
$$

It is more convenient to write the error system representation

$$
\begin{aligned}
& \dot{e}_{1}=-e_{1}+e_{2} \\
& \dot{e}_{2}=-e_{1}-e_{2}+e_{3} \\
& \dot{e}_{3}=c \tau_{\psi} R-\frac{\left(e_{3}-2 e_{2}\right)^{2}\left(e_{2}-e_{1}-k_{\omega}\right)}{V^{2}-\left(e_{2}-e_{1}-k_{\omega}\right)^{2}}-2 e_{2}-2 e_{1}+2 e_{3}
\end{aligned}
$$

Introducing $V_{L}=\frac{1}{2} e_{1}^{2}+\frac{1}{2} e_{2}^{2}+\frac{1}{2} e_{3}^{2}$ as the Lyapunov function, then

$$
\dot{V}_{L}=-e_{1}^{2}-e_{2}^{2}+e_{3}\left(e_{2}+\dot{e}_{3}\right)
$$

Let us propose the control input as

$$
c \tau_{\psi}=\frac{\left(e_{3}-2 e_{2}\right)^{2}\left(e_{2}-e_{1}-k_{\omega}\right)}{\left[V^{2}-\left(e_{2}-e_{1}-k_{\omega}\right)^{2}\right] R}+\frac{e_{2}-3 e_{3}+2 e_{1}}{R}
$$

Using (24), the control law takes the form

$$
c \tau_{\psi}=-3 r-\frac{5 e_{2}-2 e_{1}-r^{2}\left(e_{2}-e_{1}-k_{\omega}\right)}{R}
$$

Thus, (28) becomes

$$
\dot{V}_{L}=-e_{1}^{2}-e_{2}^{2}-e_{3}^{2}
$$

which proves that in the $\left(d, e_{1}, e_{2}\right)$ coordinates the equilibrium $(0,0,0)$ is GAS. In view of $(d, \psi, r)$, the resulting control is

$$
c \tau_{\psi}=-3 r+\tan \psi\left(r^{2}-5\right)-\frac{3 d+5 k_{\omega}}{V \cos \psi}
$$

